\title{
Degenerate ordered Bell numbers and polynomials associated with umbral calculus
}

\author{
Taekyun Kimª,b,*, Dae San Kimc, Gwan-Woo Jang ${ }^{b}$, Lee-Chae Jang ${ }^{d}$ \\ ${ }^{a}$ Department of Mathematics, College of Science Tianjin Polytechnic University, Tianjin 300160, China. \\ ${ }^{b}$ Department of Mathematics, Kwangwoon University, Seoul 139-701, Republic of Korea. \\ ${ }^{c}$ Department of Mathematics, Sogang University, Seoul 121-742, Republic of Korea. \\ ${ }^{d}$ Graduate School of Education, Konkuk University, Seoul 143-701, Republic of Korea.
}

Communicated by R. Saadati

\begin{abstract}
In this paper, we study degenerate ordered Bell polynomials with the viewpoint of Carlitz's degenerate Bernoulli and Euler polynomials and derive by using umbral calculus some properties and new identities for the degenerate ordered Bell polynomials associated with special polynomials. (C)2017 All rights reserved.
\end{abstract}

Keywords: Degenerate ordered Bell polynomial, umbral calculus. 2010 MSC: 11B68, 11B83, 05A40.

\section{Introduction}

As is well-known, the ordinary Euler polynomials are defined by the generating function

$$
\frac{2}{e^{t}+1} e^{x t}=\sum_{n=0}^{\infty} E_{n}(x) \frac{t^{n}}{n}, \quad(\text { see }[6-8]) .
$$

When $x=0, E_{n}=E_{n}(0)(n \geqslant 0)$ are called the Euler numbers. The Bernoulli polynomials are also given by the generating function as follows:

$$
\frac{t}{e^{t}-1} e^{x t}=\sum_{n=0}^{\infty} B_{n}(x) \frac{t^{n}}{n !}, \quad(\text { see }[4,6,7]) .
$$

When $x=0, B_{n}=B_{n}(0)$ are called the Bernoulli numbers. For $\lambda \in \mathbb{R}$, Carlitz considered the degenerate Euler and Bernoulli polynomials which are given by the generating function

$$
\frac{2}{(1+\lambda t)^{\frac{1}{\lambda}}+1}(1+\lambda t)^{\frac{x}{\lambda}}=\sum_{n=0}^{\infty} \varepsilon_{n}(x \mid \lambda) \frac{t^{n}}{n !},
$$

\footnotetext{
*Corresponding author

Email addresses: tkkim@kw.ac.kr (Taekyun Kim), dskim@sogang.ac.kr (Dae San Kim), gwjang@kw.ac.kr (Gwan-Woo Jang), lcjang@konkuk.ac.kr (Lee-Chae Jang)

doi:10.22436/jnsa.010.10.02
} 
and

$$
\frac{t}{(1+\lambda t)^{\frac{1}{\lambda}}-1}(1+\lambda t)^{\frac{x}{\lambda}}=\sum_{n=0}^{\infty} \beta_{n}(x \mid \lambda) \frac{t^{n}}{n !}, \quad \text { (see [2]). }
$$

Note that $\lim _{\lambda \rightarrow 0} \varepsilon_{n}(x \mid \lambda)=E_{n}(x)$ and $\lim _{\lambda \rightarrow 0} \beta_{n}(x \mid \lambda)=B_{n}(x)(n \geqslant 0)$, (see $\left.[2,8]\right)$. The falling factorial sequences are defined by

$$
(x)_{0}=1,(x)_{n}=x(x-1) \cdots(x-(n-1)),(n \geqslant 1) .
$$

The Stirling numbers of the first kind are defined as

$$
(x)_{n}=\sum_{l=0}^{n} S_{1}(n, l) x^{l}, \quad(n \geqslant 0), \quad(\text { see }[10]) .
$$

The Stirling numbers of the second kind are also defined by

$$
x^{n}=\sum_{l=0}^{n} S_{2}(n, l)(x)_{l}, \quad(n \geqslant 0), \quad(\text { see }[6,10]) .
$$

It is well-known that the ordered Bell polynomials are defined by the generating function

$$
\frac{1}{2-e^{t}} e^{x t}=\sum_{n=0}^{\infty} b_{n}(x) \frac{t^{n}}{n !}, \quad(\text { see }[3]) .
$$

When $x=0, b_{n}=b_{n}(0)$ are called the ordered Bell numbers. From (1.3), we note that

$$
\begin{aligned}
\frac{1}{2-e^{t}} & =\frac{1}{1-\left(e^{t}-1\right)}=\sum_{m=0}^{\infty}\left(e^{t}-1\right)^{m} \\
& =\sum_{m=0}^{\infty} m ! \sum_{n=m}^{\infty} S_{2}(n, m) \frac{t^{n}}{n !}=\sum_{n=0}^{\infty}\left(\sum_{m=0}^{n} m ! S_{2}(n, m)\right) \frac{t^{n}}{n !} .
\end{aligned}
$$

Thus, by (1.4), we get

$$
b_{n}=\sum_{m=0}^{n} m ! S_{2}(n, m),(n \geqslant 0) .
$$

For $r \in \mathbb{N}$, the higher-order ordered Bell polynomials are given by the generating function

$$
\left(\frac{1}{2-e^{t}}\right)^{r} e^{x t}=\sum_{n=0}^{\infty} b_{n}^{(r)}(x) \frac{t^{n}}{n !}, \quad(\text { see [3] ). }
$$

When $x=0, b_{n}^{(r)}=b_{n}^{(r)}(0)(n \geqslant 0)$ are called the higher-order ordered Bell numbers. By (1.5), we easily get

$$
\begin{aligned}
\sum_{n=0}^{\infty} b_{n}^{(r)} \frac{t^{n}}{n !} & =\left(2-e^{t}\right)^{-r}=\left(1-\left(e^{t}-1\right)\right)^{-r} \\
& =\sum_{m=0}^{\infty}\left(\begin{array}{c}
m+r-1 \\
m
\end{array}\right)\left(e^{t}-1\right)^{m} \\
& =\sum_{m=0}^{\infty}\left(\begin{array}{c}
m+r-1 \\
m
\end{array}\right) m ! \sum_{n=m}^{\infty} S_{2}(n, m) \frac{t^{n}}{n !} \\
& =\sum_{n=0}^{\infty}\left(\sum_{m=0}^{n}\left(\begin{array}{c}
m+r-1 \\
m
\end{array}\right) m ! S_{2}(n, m)\right) \frac{t^{n}}{n !}
\end{aligned}
$$


From (1.6), we note that

$$
b_{n}^{(r)}=\sum_{m=0}^{n}\left(\begin{array}{c}
m+r-1 \\
m
\end{array}\right) m ! S_{2}(n, m),(n \geqslant 0) .
$$

In view of (1.1) and (1.2), we consider the degenerate ordered Bell polynomials given by the generating function

$$
\frac{1}{2-(1+\lambda t)^{\frac{1}{\lambda}}}(1+\lambda t)^{\frac{x}{\lambda}}=\sum_{n=0}^{\infty} b_{n, \lambda}(x) \frac{t^{n}}{n !} .
$$

By (1.7), we easily get

$$
\lim _{\lambda \rightarrow 0} b_{n, \lambda}(x)=b_{n}(x),(n \geqslant 0) .
$$

When $x=0, b_{n, \lambda}=b_{n, \lambda}(0)$ are called the degenerate ordered Bell numbers. Replacing $t$ by $\frac{1}{\lambda}\left(e^{\lambda t-1}\right)$, we get

$$
\begin{aligned}
\sum_{m=0}^{\infty} b_{m, \lambda}(x) \frac{1}{m !}\left(\frac{1}{\lambda}\left(e^{\lambda t-1}\right)\right)^{m} & =\frac{1}{2-e^{t}} e^{x t} \\
& =\sum_{n=0}^{\infty} b_{n}(x) \frac{t^{n}}{n !} .
\end{aligned}
$$

Now, we observe that

$$
\begin{aligned}
\sum_{m=0}^{\infty} b_{m, \lambda}(x) \frac{1}{m !} \lambda^{-m}\left(e^{\lambda t}-1\right)^{m} & =\sum_{m=0}^{\infty} b_{m, \lambda}(x) \lambda^{-m} \sum_{n=m}^{\infty} S_{2}(n, m) \lambda^{n} \frac{t^{n}}{n !} \\
& =\sum_{n=0}^{\infty}\left(\sum_{m=0}^{n} \lambda^{n-m} b_{m, \lambda}(x) S_{2}(n, m)\right) \frac{t^{n}}{n !}
\end{aligned}
$$

From (1.8) and (1.9), we have

$$
b_{n}(x)=\sum_{m=0}^{n} \lambda^{n-m} b_{m, \lambda}(x) S_{2}(n, m) .
$$

We study degenerate ordered Bell polynomials with the viewpoint of Carlitz's degenerate Bernoulli and Euler polynomials and derive by using umbral calculus some properties and new identities for the degenerate ordered Bell polynomials associated with special polynomials.

\section{Quick review of umbral calculus}

Let $\mathbb{C}$ be the complex number field and let $\mathcal{F}$ be the set of all formal power series with coefficients in $\mathbb{C}$ in the variable $t$

$$
\mathcal{F}=\left\{f(t)=\sum_{k=0}^{\infty} a_{k} \frac{t^{k}}{k !} \mid a_{k} \in \mathbb{C}\right\} .
$$

We denote the algebra $\mathbb{C}[x]$ of polynomials in $x$ over the field $\mathbb{C}$ by $\mathbb{P}(\mathbb{P}=\mathbb{C}[x])$. Let $\mathbb{P}^{*}$ be the vector space of all linear functionals on $\mathbb{P}$, and let $<L \mid p(x)>$ denote the action of the linear functional $L$ on $\mathrm{p}(\mathrm{x})$, which satisfies $<\mathrm{L}+\mathrm{M}|\mathrm{p}(\mathrm{x})>=<\mathrm{L}| \mathrm{p}(\mathrm{x})>+<\mathrm{M} \mid \mathrm{p}(\mathrm{x})>$ and $<\mathrm{cL}|\mathrm{p}(\mathrm{x})>=\mathrm{c}<\mathrm{L}| \mathrm{p}(\mathrm{x})>$, where $\mathrm{c}$ 
is a complex number. The linear functional $<f(t) \mid \cdot>$ on $\mathbb{P}$ is defined by $<f(t) \mid x^{n}>=a_{n}(n \geqslant 0)$, where $f(t)=\sum_{k=0}^{\infty} a_{k} \frac{t^{k}}{k !} \in \mathcal{F}$. Thus we note that $<t^{k} \mid x^{n}>=n ! \delta_{n, k}(n, k \geqslant 0)$, where $\delta_{n, k}$ is the Kronecker symbol (see $[1,5,10])$. Let $f_{L}(t)=\sum_{k=0}^{\infty} \frac{<L \mid x^{k}>}{k !} t^{k}$. Then we have $<f_{L}(t)\left|x^{n}>=<L\right| x^{n}>(n \geqslant 0)$. So, the map $L \longmapsto f_{L}(t)$ is a vector space isomorphism from $\mathbb{P}^{*}$ onto $\mathcal{F}$. Henceforth, $\mathcal{F}$ denotes both the algebra of formal power series in $t$ and the vector space of all linear functionals on $\mathbb{P}$, and so an element $f(t)$ of $\mathcal{F}$ will be thought of as both a formal power series and a linear functional. We call $\mathcal{F}$ the umbral algebra. The umbral calculus is the study of the umbral algebra. Let $f(t)(\neq 0) \in \mathcal{F}$. Then order of $f(t)$ is the smallest positive integer $k$ for which the coefficient of $t^{k}$ does not vanish. The order of $f(t)$ is denoted by $o(f(t))$, (see $[7,8,10])$. For $f(t) \in \mathcal{F}$ and $p(x) \in \mathbb{P}$, we have

$$
f(t)=\sum_{k=0}^{\infty}<f(t)\left|x^{k}>\frac{t^{k}}{k !}, \quad p(x)=\sum_{k=0}^{\infty}<t^{k}\right| p(x)>\frac{t^{k}}{k !} .
$$

Thus, by (2.1), we easily get

$$
p^{(k)}(0)=<t^{k}|p(x)>=<1| p^{(k)}(x)>, \quad(\text { see }[9,10]),
$$

where $p^{(k)}(x)=\left(\frac{d}{d x}\right)^{k} p(x)$. From (2.2), we note that

$$
t^{k} p(x)=p^{(k)}(x), e^{y t} p(x)=p(x+y), \text { and }<e^{y t} \mid p(x)>=p(y), \quad(\text { see [10-12]). }
$$

For $f(t), g(t) \in \mathcal{F}$ with $o(f(t))=1, o(g(t))=0$, there exists a unique sequence $S_{n}(x)$ of polynomials with $\operatorname{deg} S_{\mathfrak{n}}(x)=n$ such that

$$
<g(t) f(t)^{k} \mid S_{n}(x)>=n ! \delta_{n, k},(n, k \geqslant 0), \quad(\text { see }[6,8,10]) .
$$

The sequence $S_{n}(x)$ is called the Sheffer sequence for $(g(t), f(t))$, and we write $S_{n}(x) \sim(g(t), f(t))$. It is well-known that

$$
S_{n}(x) \sim(g(t), f(t)) \Longleftrightarrow \frac{1}{g(\bar{f}(t))} e^{x \bar{f}(t)}=\sum_{n=0}^{\infty} S_{n}(x) \frac{t^{n}}{n !},
$$

where $\bar{f}(t)$ is the compositional inverse of $f(t)$ such that $f(\bar{f}(t))=\bar{f}(f(t))=t$. Let $S_{\mathfrak{n}}(x) \sim(g(t), f(t))$. Then we have

$$
\begin{gathered}
f(t) s_{n}(x)=n S_{n-1}(x), \quad(n \geqslant 1), \quad S_{n}(x)=\sum_{j=0}^{n} \frac{<g(\bar{f}(t))^{-1} \bar{f}(t)^{j} \mid x^{n}>}{j !} x^{j}, \\
S_{n}(x+y)=\sum_{j=0}^{n}\left(\begin{array}{l}
n \\
j
\end{array}\right) S_{j}(x) P_{n-j}(y), \text { where } P_{n}(y)=g(t) S_{n}(y), \\
<f(t)\left|x p(x)>=<\delta_{t} f(t)\right| p(x)>, \text { where } \delta_{t} f(t)=\frac{d}{d t} f(t),
\end{gathered}
$$

and

$$
S_{n+1}(x)=\left(x-\frac{g^{\prime}(t)}{g(t)}\right) \frac{1}{f^{\prime}(t)} S_{n}(x),(n \geqslant 0), \quad(\text { see }[10])
$$

For $p_{n}(x) \sim(1, f(t)), q_{n}(x) \sim(1, g(t))$, we have

$$
q_{n}(x)=x\left(\frac{f(t)}{g(t)}\right)^{n} x^{-1} p_{n}(x), \quad(n \geqslant 1), \quad(\text { see }[10,11]) .
$$


Let us consider the following two Sheffer sequences:

$$
S_{n}(x) \sim(g(t), f(t)), \quad r_{n}(x) \sim(h(t), l(t)) .
$$

Then we have

$$
S_{n}(x)=\sum_{m=0}^{n} C_{n, m} r_{m}(x),(n \geqslant 0),
$$

where

$$
C_{n, m}=\frac{1}{m !}\left\langle\frac { h ( \overline { f } ( t ) ) } { g ( \overline { f } ( t ) ) } \left( l((\bar{f}(t)))^{m}\left|x^{n}\right\rangle, \quad(\text { see }[10])\right.\right.
$$

Finally, we recall that

$$
\frac{d}{d x} S_{n}(x)=\sum_{k=0}^{n-1}\left(\begin{array}{l}
n \\
k
\end{array}\right)<\bar{f}(t) \mid x^{n-k}>S_{k}(x) .
$$

\section{Degenerate ordered Bell numbers and polynomials associated with umbral calculus}

From (1.7), we note that the degenerate ordered Bell polynomials are defined by the generating function

$$
\frac{1}{2-(1+\lambda t)^{\frac{1}{\lambda}}}(1+\lambda t)^{\frac{x}{\lambda}}=\sum_{n=0}^{\infty} b_{n, \lambda}(x) \frac{t^{n}}{n !} .
$$

When $x=0, b_{n, \lambda}=b_{n, \lambda}(0)$ are called the ordered Bell numbers. By (1.7), we get

$$
\begin{aligned}
\sum_{n=0}^{\infty} b_{n, \lambda} \frac{t^{n}}{n !} & =\frac{1}{2-(1+\lambda t)^{\frac{1}{\lambda}}}=\frac{1}{1-\left((1+\lambda t)^{\frac{1}{\lambda}}-1\right)} \\
& =\sum_{m=0}^{\infty}\left((1+\lambda t)^{\frac{1}{\lambda}}-1\right)^{m}=\sum_{m=0}^{\infty}\left(e^{\frac{1}{\lambda} \log (1+\lambda t)}-1\right)^{m} \\
& =\sum_{m=0}^{\infty} m ! \sum_{k=m}^{\infty} S_{2}(k, m) \lambda^{-k} \frac{1}{k !}(\log (1+\lambda t))^{k} \\
& =\sum_{k=0}^{\infty}\left(\sum_{m=0}^{k} m ! S_{2}(k, m) \lambda^{-k}\right) \sum_{n=k}^{\infty} S_{1}(n, k) \frac{\lambda^{n} t^{n}}{n !} \\
& =\sum_{n=0}^{\infty}\left(\sum_{k=0}^{n} \sum_{m=0}^{k} m ! S_{2}(k, m) S_{1}(n, k) \lambda^{n-k}\right) \frac{t^{n}}{n !} .
\end{aligned}
$$

From (3.1), we note that

$$
b_{n, \lambda}=\sum_{k=0}^{n} \sum_{m=0}^{k} m ! S_{2}(k, m) S_{1}(n, k) \lambda^{n-k},(n \geqslant 0) .
$$

By (1.7) and (2.3), we get

$$
b_{n, \lambda}(x) \sim\left(2-e^{t}, \frac{1}{\lambda}\left(e^{\lambda t}-1\right)\right),(n \geqslant 0)
$$

That is,

$$
\frac{1}{2-e^{\frac{1}{\lambda} \log (1+\lambda t)}} e^{x \frac{1}{\lambda} \log (1+\lambda t)}=\sum_{n=0}^{\infty} b_{n, \lambda}(x) \frac{t^{n}}{n !} .
$$


Let $f(t)$ be the linear functional such that

$$
<\mathrm{f}(\mathrm{t}) \mid \mathrm{p}(\mathrm{x})>=\int_{0}^{y} \mathrm{p}(\mathrm{u}) \mathrm{du}
$$

for all $p(x)$. Then, by (2.1) and (3.4), we get

$$
\begin{aligned}
f(t) & =\sum_{k=0}^{\infty} \frac{<f(t) \mid x^{k}>}{k !} t^{k}=\sum_{k=0}^{\infty} \frac{y^{k+1}}{(k+1) !} t^{k} \\
& =\frac{1}{t}\left(e^{y t}-1\right) .
\end{aligned}
$$

From (3.4), we have

$$
<\frac{1}{\mathfrak{t}}\left(e^{y t}-1\right) \mid p(x)>=\int_{0}^{y} p(u) d u
$$

and

$$
\frac{1}{t}\left(e^{y t}-1\right) p(x)=\int_{x}^{x+y} p(u) d u
$$

By (3.1), (3.3), we get

$$
\begin{aligned}
\sum_{n=0}^{\infty} b_{n, \lambda}(x) \frac{t^{n}}{n !} & =\frac{1}{2-(1+\lambda t)^{\frac{1}{\lambda}}} e^{\frac{x}{\lambda} \log (1+\lambda t)} \\
& =\left(\sum_{l=0}^{\infty} b_{l, \lambda} \frac{t^{l}}{l !}\right)\left(\sum_{m=0}^{\infty}\left(\frac{x}{\lambda}\right)^{m} \frac{1}{m !}(\log (1+\lambda t))^{m}\right) \\
& =\left(\sum_{l=0}^{\infty} b_{l, \lambda} \frac{t^{l}}{l !}\right)\left(\sum_{m=0}^{\infty}\left(\frac{x}{\lambda}\right)^{m} \sum_{k=m}^{\infty} S_{1}(k, m) \frac{\lambda^{k} t^{k}}{k !}\right) \\
& =\left(\sum_{l=0}^{\infty} b_{l, \lambda} \frac{t^{l}}{l !}\right)\left(\sum_{k=0}^{\infty}\left(\sum_{m=0}^{k} \lambda^{k-m} S_{1}(k, m) x^{m}\right) \frac{t^{k}}{k !}\right) \\
& =\sum_{n=0}^{\infty}\left\{\sum_{k=0}^{n} \sum_{m=0}^{k} \lambda^{k-m} S_{1}(k, m)\left(\begin{array}{l}
n \\
k
\end{array}\right) x^{m} b_{n-k, \lambda}\right\} \frac{t^{n}}{n !} .
\end{aligned}
$$

Comparing the coefficients on both sides of (3.6), we have

$$
b_{n, \lambda}(x)=\sum_{k=0}^{n} \sum_{m=0}^{k}\left(\begin{array}{l}
n \\
k
\end{array}\right) \lambda^{k-m} S_{1}(k, m) b_{n-k, \lambda} x^{m} .
$$

From (3.5), we note that

$$
\begin{aligned}
\frac{e^{t}-1}{t} b_{n, \lambda}(x) & =\int_{x}^{x+1} b_{n, \lambda}(u) d u \\
& =\sum_{k=0}^{n} \sum_{m=0}^{k}\left(\begin{array}{l}
n \\
k
\end{array}\right) \lambda^{k-m} S_{1}(k, m) b_{n-k, \lambda} \int_{x}^{x+1} u^{m} d u \\
& =\sum_{k=0}^{n} \sum_{m=0}^{k}\left(\begin{array}{l}
n \\
k
\end{array}\right) \lambda^{k-m} S_{1}(k, m) b_{n-k, \lambda} \frac{1}{m+1}\left((x+1)^{m+1}-x^{m+1}\right) \\
& =\sum_{k=0}^{n} \sum_{m=0}^{k} \sum_{l=0}^{m}\left(\begin{array}{c}
m+1 \\
l
\end{array}\right)\left(\begin{array}{l}
n \\
k
\end{array}\right) \lambda^{k-m} S_{1}(k, m) b_{n-k, \lambda} \frac{1}{m+1} x^{l}
\end{aligned}
$$


As is well-known, $B_{n}(x)=\frac{t}{e^{t}-1} x^{n},(n \geqslant 0)$. By (3.8), we get

$$
b_{n, \lambda}(x)=\sum_{k=0}^{n} \sum_{m=0}^{k} \sum_{l=0}^{m} \lambda^{k-m}\left(\begin{array}{c}
m+1 \\
l
\end{array}\right)\left(\begin{array}{l}
n \\
k
\end{array}\right) s_{1}(k, m) b_{n-k, \lambda} \frac{1}{m+1} B_{l}(x) .
$$

From (3.9), we note that

$$
\begin{aligned}
\operatorname{tb}_{n, \lambda}(x) & =\sum_{k=1}^{n} \sum_{m=1}^{k} \sum_{l=1}^{m} \lambda^{k-m}\left(\begin{array}{c}
m+1 \\
l
\end{array}\right)\left(\begin{array}{l}
n \\
k
\end{array}\right) \frac{l}{m+1} B_{l-1}(x) S_{1}(k, m) b_{n-k, \lambda} \\
& =\sum_{k=1}^{n} \sum_{m=1}^{k} \sum_{l=1}^{m} \lambda^{k-m}\left(\begin{array}{c}
m \\
l-1
\end{array}\right)\left(\begin{array}{l}
n \\
k
\end{array}\right) B_{l-1}(x) S_{1}(k, m) b_{n-k, \lambda}
\end{aligned}
$$

and

$$
\begin{aligned}
\frac{e^{y t}-1}{t} & b_{n, \lambda}(x) \\
& =\int_{x}^{x+y} b_{n, \lambda}(u) d u \\
& =\sum_{k=0}^{n} \sum_{m=0}^{k} \sum_{l=0}^{m} \lambda^{k-m}\left(\begin{array}{c}
m+1 \\
l
\end{array}\right)\left(\begin{array}{l}
n \\
k
\end{array}\right) s_{1}(k, m) b_{n-k, \lambda}\left(\frac{B_{l+1}(x+y)-B_{l+1}(x)}{(m+1)(l+1)}\right) \\
& =\sum_{k=0}^{n} \sum_{m=0}^{k} \sum_{l=0}^{m} \sum_{j=0}^{l}\left(\begin{array}{c}
m+1 \\
l
\end{array}\right)\left(\begin{array}{c}
n \\
k
\end{array}\right)\left(\begin{array}{c}
l+1 \\
j
\end{array}\right) \lambda^{k-m} S_{1}(k, m) b_{n-k, \lambda} y^{l+1-j} B_{j}(x) \frac{1}{(m+1)(l+1)} \\
& =\sum_{k=0}^{n} \sum_{m=0}^{k} \sum_{l=0}^{m} \sum_{j=0}^{l} \frac{\left(\begin{array}{c}
m+1 \\
l
\end{array}\right)\left(\begin{array}{c}
n \\
k
\end{array}\right)\left(\begin{array}{c}
l+1 \\
j
\end{array}\right)}{(m+1)(l+1)} \lambda^{k-m} S_{1}(k, m) b_{n-k, \lambda} y^{l+1-j} B_{j}(x) .
\end{aligned}
$$

By multiplying $t$ on both sides of (3.10), we get

$$
\begin{aligned}
b_{n, \lambda}(x+y)-b_{n, \lambda}(x) & =\left(e^{y t}-1\right) b_{n, \lambda}(x) \\
& =\sum_{k=1}^{n} \sum_{m=1}^{k} \sum_{l=1}^{m} \sum_{j=1}^{l} \frac{\left(\begin{array}{c}
m+1 \\
l
\end{array}\right)\left(\begin{array}{c}
n \\
k
\end{array}\right)\left(\begin{array}{c}
l+1 \\
j
\end{array}\right) j}{(m+1)(l+1)} j \lambda^{k-m} S_{1}(k, m) b_{n-k, \lambda} y^{l+1-j} B_{j-1}(x) \\
& =\sum_{k=1}^{n} \sum_{m=1}^{k} \sum_{l=1}^{m} \sum_{j=1}^{l} \frac{\left(\begin{array}{c}
m+1 \\
l
\end{array}\right)\left(\begin{array}{c}
n \\
k
\end{array}\right)\left(\begin{array}{c}
l \\
j-1
\end{array}\right)}{m+1} \lambda^{k-m} S_{1}(k, m) b_{n-k, \lambda} y^{l+1-j} B_{j-1}(x) .
\end{aligned}
$$

Therefore, by (3.11) we obtain the following theorem.

Theorem 3.1. For $\mathrm{n} \geqslant 1$, we have

$$
b_{n, \lambda}(x+y)-b_{n, \lambda}(x)=\sum_{k=1}^{n} \sum_{m=1}^{k} \sum_{l=1}^{m} \sum_{j=1}^{l} \frac{\left(\begin{array}{c}
m+1 \\
l
\end{array}\right)\left(\begin{array}{c}
n \\
k
\end{array}\right)\left(\begin{array}{c}
l \\
j-1
\end{array}\right)}{m+1} \lambda^{k-m} S_{1}(k, m) b_{n-k, \lambda} y^{l+1-j} B_{j-1}(x) .
$$

Now, we observe that

$$
\begin{aligned}
\sum_{n=0}^{\infty}\left(-b_{n, \lambda}(x+1)+2 b_{n, \lambda}(x)\right) \frac{t^{n}}{n !} & =\frac{(1+\lambda t)^{\frac{x}{\lambda}}\left(2-(1+\lambda t)^{\frac{1}{\lambda}}\right)}{2-(1+\lambda t)^{\frac{1}{\lambda}}} \\
& =(1+\lambda t)^{\frac{x}{\lambda}}=\sum_{k=0}^{\infty}\left(\frac{x}{\lambda}\right)^{k} \frac{1}{k !}(\log (1+\lambda t))^{k} \\
& =\sum_{k=0}^{\infty}\left(\frac{x}{\lambda}\right)^{k} \sum_{n=k}^{\infty} S_{1}(n, k) \lambda^{n} \frac{t^{n}}{n !}=\sum_{n=0}^{\infty}\left(\sum_{k=0}^{n} \lambda^{n-k} S_{1}(n, k) x^{k}\right) \frac{t^{n}}{n !} .
\end{aligned}
$$


Comparing the coefficients on both sides of (3.12), we obtain the following theorem.

Theorem 3.2. For $n \geqslant 0$, we have

$$
2 b_{n, \lambda}(x)-b_{n, \lambda}(x+1)=\sum_{k=0}^{n} \lambda^{n-k} S_{1}(n, k) x^{k} .
$$

From Theorem 3.1 and Theorem 3.2, we note that

$$
\begin{aligned}
b_{n, \lambda}(x+1)-b_{n, \lambda}(x) & =b_{n, \lambda}(x)-\sum_{k=0}^{n} \lambda^{n-k} S_{1}(n, k) x^{k} \\
& =\sum_{k=1}^{n} \sum_{m=1}^{k} \sum_{l=1}^{m} \sum_{j=1}^{l} \frac{\left(\begin{array}{c}
m+1 \\
l
\end{array}\right)\left(\begin{array}{c}
n \\
k
\end{array}\right)\left(\begin{array}{c}
l \\
j-1
\end{array}\right)}{m+1} \lambda^{k-m} S_{1}(k, m) b_{n-k, \lambda} B_{j-1}(x) .
\end{aligned}
$$

Therefore, by (3.13), we obtain the following corollary.

Corollary 3.3. For $n \geqslant 1$, we have

$$
\begin{aligned}
b_{n, \lambda}(x)= & \sum_{k=1}^{n} \lambda^{n-k} S_{1}(n, k) x^{k} \\
& +\sum_{k=1}^{n} \sum_{m=1}^{k} \sum_{l=1}^{m} \sum_{j=1}^{l} \frac{\left(\begin{array}{c}
m+1 \\
l
\end{array}\right)\left(\begin{array}{c}
n \\
k
\end{array}\right)\left(\begin{array}{c}
l \\
j-1
\end{array}\right)}{m+1} \lambda^{k-m} S_{1}(k, m) b_{n-k, \lambda} B_{j-1}(x) .
\end{aligned}
$$

From $<t^{k} \mid x^{n}>=n ! \delta_{n, k},(n, k \geqslant 0)$, we note that

$$
\begin{aligned}
b_{n, \lambda}(y) & =\left\langle\sum_{l=0}^{\infty} b_{l, \lambda}(y) \frac{t^{l}}{l !} \mid x^{n}\right\rangle=\left\langle\frac{1}{2-(1+\lambda t)^{\frac{1}{\lambda}}}(1+\lambda t)^{\frac{y}{\lambda}} \mid x^{n}\right\rangle \\
& =\left\langle\frac{1}{2-(1+\lambda t)^{\frac{1}{\lambda}}} \mid(1+\lambda t)^{\frac{y}{\lambda}} x^{n}\right\rangle \\
& =\sum_{m=0}^{n}\left(\begin{array}{c}
n \\
m
\end{array}\right) \sum_{k=0}^{m} y^{k} \lambda^{m-k} S_{1}(m, k)\left\langle\frac{1}{2-(1+\lambda t)^{\frac{1}{\lambda}}} \mid x^{n-m}\right\rangle \\
& =\sum_{m=0}^{n}\left(\begin{array}{c}
n \\
m
\end{array}\right) \sum_{k=0}^{m} y^{k} \lambda^{m-k} S_{1}(m, k) \sum_{l=0}^{n-m} \frac{b_{l, \lambda}}{l !}<t^{l}\left|x^{n-m}\right\rangle \\
& =\sum_{m=0}^{n} \sum_{k=0}^{m}\left(\begin{array}{c}
n \\
m
\end{array}\right) \lambda^{m-k} S_{1}(m, k) y^{k} b_{n-m, \lambda} .
\end{aligned}
$$

Therefore, by (3.14), we obtain the following theorem.

Theorem 3.4. For $\mathrm{n} \geqslant 0$, we have

$$
b_{n, \lambda}(x)=\sum_{m=0}^{n} \sum_{k=0}^{m}\left(\begin{array}{l}
n \\
m
\end{array}\right) \lambda^{m-k} S_{1}(m, k) b_{n-m, \lambda} x^{k} .
$$

We easily see that

$$
x^{\mathfrak{n}} \sim(1, t),\left(2-e^{t}\right) b_{n, \lambda}(x) \sim\left(1, \frac{1}{\lambda}\left(e^{\lambda t}-1\right)\right) .
$$


Then, by (2.5) and (3.15) we get

$$
\begin{aligned}
\left(2-e^{t}\right) b_{n, \lambda}(x) & =x\left(\frac{\lambda t}{e^{\lambda t}-1}\right)^{n} x^{-1} x^{n} \\
& =x \sum_{l=0}^{\infty} \lambda^{l} \frac{B_{l}^{(n)}}{l !} t^{l} x^{n-1} \\
& =x \sum_{l=0}^{n-1} \lambda^{l}\left(\begin{array}{c}
n-1 \\
l
\end{array}\right) B_{l}^{(n)} x^{n-1-l}=\sum_{l=0}^{n-1} \lambda^{l}\left(\begin{array}{c}
n-1 \\
l
\end{array}\right) B_{l}^{(n)} x^{n-l},
\end{aligned}
$$

where $B_{n}^{(\alpha)}$ are the higher-order Bernoulli numbers defined by the generating function

$$
\left(\frac{t}{e^{t}-1}\right)^{\alpha}=\sum_{n=0}^{\infty} B_{n}^{(\alpha)} \frac{t^{n}}{n !} \text {. }
$$

Thus, by (3.16) we get

$$
\begin{aligned}
b_{n, \lambda}(x) & =\sum_{l=0}^{n-1} \lambda^{l}\left(\begin{array}{c}
n-1 \\
l
\end{array}\right) B_{l}^{(n)} \frac{1}{2-e^{t}} x^{n-l} \\
& =\sum_{l=0}^{n-1} \lambda^{l}\left(\begin{array}{c}
n-1 \\
l
\end{array}\right) B_{l}^{(n)} b_{n-l}(x),(n \geqslant 1) .
\end{aligned}
$$

Therefore, by (3.17), we obtain the following theorem.

Theorem 3.5. For $\mathrm{n} \geqslant 1$, we have

$$
\begin{aligned}
b_{n, \lambda}(x) & =\sum_{l=0}^{n-1} \lambda^{l}\left(\begin{array}{c}
n-1 \\
l
\end{array}\right) B_{l}^{(n)} b_{n-l, \lambda}(x) \\
& =\sum_{l=0}^{n} \sum_{m=0}^{n-l} \lambda^{l}\left(\begin{array}{c}
n-1 \\
l
\end{array}\right)\left(\begin{array}{c}
n-l \\
m
\end{array}\right) B_{l}^{(n)} b_{m} x^{n-l-m} .
\end{aligned}
$$

Let $S_{\mathfrak{n}}(x) \sim\left(1, \frac{1}{\lambda}\left(e^{\lambda t}-1\right)\right)$. Then, by (2.3) we get

$$
\begin{aligned}
\sum_{n=0}^{\infty} S_{n}(x) \frac{t^{n}}{n !} & =e^{\frac{x}{\lambda} \log (1+\lambda t)}=(1+\lambda t)^{\frac{x}{\lambda}} \\
& =\sum_{n=0}^{\infty}\left(\frac{x}{\lambda}\right)_{n} \lambda^{n} \frac{t^{n}}{n !}=\sum_{n=0}^{\infty} \frac{x}{\lambda}\left(\frac{x}{\lambda}-1\right) \cdots\left(\frac{x}{\lambda}-n+1\right) \lambda^{n} \frac{t^{n}}{n !} \\
& =\sum_{n=0}^{\infty} x(x-\lambda)(x-2 \lambda) \cdots(x-(n-1) \lambda) \frac{t^{n}}{n !} \\
& =\sum_{n=0}^{\infty}(x)_{n, \lambda} \frac{t^{n}}{n !},
\end{aligned}
$$

where $(x)_{0, \lambda}=1,(x)_{n, \lambda}=x(x-\lambda)(x-2 \lambda) \cdots(x-(n-1) \lambda),(n \geqslant 1)$. From (3.18), we note that $(x)_{n, \lambda} \sim$ $\left(1, \frac{1}{\lambda}\left(e^{\lambda t}-1\right)\right)$. Now, we observe that

$$
\begin{aligned}
\sum_{n=0}^{\infty}(x)_{n, \lambda} \frac{t^{n}}{n !} & =(1+\lambda t)^{\frac{x}{\lambda}}=e^{\frac{x}{\lambda} \log (1+\lambda t)} \\
& =\sum_{m=0}^{\infty}\left(\frac{x}{\lambda}\right)^{m} \frac{1}{m !}(\log (1+\lambda t))^{m} \\
& =\sum_{n=0}^{\infty}\left(\sum_{m=0}^{n} \lambda^{n-m} S_{1}(n, m) x^{m}\right) \frac{t^{n}}{n !} .
\end{aligned}
$$


Comparing the coefficients on both sides of (3.19), we have

$$
(x)_{n, \lambda}=\sum_{m=0}^{n} \lambda^{n-m} S_{1}(n, m) x^{m},(n \geqslant 0) .
$$

For $\left(2-e^{t}\right) b_{n, \lambda}(x) \sim\left(1, \frac{1}{\lambda}\left(e^{\lambda t}-1\right)\right),(x)_{n, \lambda} \sim\left(1, \frac{1}{\lambda}\left(e^{\lambda t}-1\right)\right)$, we get

$$
\left(2-e^{t}\right) b_{n, \lambda}(x)=(x)_{n, \lambda}=\sum_{m=0}^{n} \lambda^{n-m} S_{1}(n, m) x^{m} .
$$

By (3.20), we easily see that

$$
\begin{aligned}
b_{n, \lambda}(x) & =\sum_{m=0}^{n} \lambda^{n-m} S_{1}(n, m) \frac{1}{2-e^{t}} x^{m} \\
& =\sum_{m=0}^{n} \lambda^{n-m} S_{1}(n, m) b_{m}(x),(n \geqslant 0) .
\end{aligned}
$$

Corollary 3.6. For $\mathrm{n} \geqslant 0$, we have

$$
b_{n, \lambda}(x)=\sum_{m=0}^{n} \lambda^{n-m} S_{1}(n, m) b_{m}(x)
$$

By (2.4) and (3.2), we get

$$
\begin{aligned}
b_{n+1, \lambda}(x) & =\left(x-\frac{\left(-e^{t}\right)}{2-e^{t}}\right) \frac{1}{e^{\lambda t}} b_{n, \lambda}(x) \\
& =\left(x+\frac{e^{t}}{2-e^{t}}\right) b_{n, \lambda}(x-\lambda)=x b_{n, \lambda}(x-\lambda)+\frac{e^{t}}{2-e^{t}} b_{n, \lambda}(x-\lambda) \\
& =x b_{n, \lambda}(x-\lambda)+\frac{e^{t}-2+2}{2-e^{t}} b_{n, \lambda}(x-\lambda) \\
& =x b_{n, \lambda}(x-\lambda)-b_{n, \lambda}(x-\lambda)+\frac{2}{2-e^{t}} b_{n, \lambda}(x-\lambda) .
\end{aligned}
$$

From (3.7), we note that

$$
\begin{aligned}
\frac{1}{2-e^{\mathrm{t}}} b_{n, \lambda}(x-\lambda) & =\frac{1}{2-e^{\mathrm{t}}} \sum_{k=0}^{n} \sum_{m=0}^{k}\left(\begin{array}{l}
n \\
k
\end{array}\right) \lambda^{k-m} S_{1}(k, m) b_{n-k, \lambda}(x-\lambda)^{m} \\
& =\frac{1}{2-e^{\mathrm{t}}} \sum_{k=0}^{n} \sum_{m=0}^{k}\left(\begin{array}{l}
n \\
k
\end{array}\right) \lambda^{k-m} S_{1}(k, m) b_{n-k, \lambda} \sum_{l=0}^{m}\left(\begin{array}{c}
m \\
l
\end{array}\right)(-1)^{l} \lambda^{l} x^{m-l} \\
& =\sum_{k=0}^{n} \sum_{m=0}^{k} \sum_{l=0}^{m}\left(\begin{array}{l}
n \\
k
\end{array}\right)\left(\begin{array}{c}
m \\
l
\end{array}\right) \lambda^{k-m+l} S_{1}(k, m) b_{n-k, \lambda}(-1)^{l} \frac{1}{2-e^{t}} x^{m-l} \\
& =\sum_{k=0}^{n} \sum_{m=0}^{k} \sum_{l=0}^{m}\left(\begin{array}{l}
n \\
k
\end{array}\right)\left(\begin{array}{c}
m \\
l
\end{array}\right) \lambda^{k-m+l} S_{1}(k, m) b_{n-k, \lambda}(-1)^{l} b_{m-l}(x) .
\end{aligned}
$$

By (3.21) and (3.22), we get

$$
\begin{aligned}
b_{n+1, \lambda}(x)= & x b_{n, \lambda}(x-\lambda)-b_{n, \lambda}(x-\lambda)+\frac{2}{2-e^{t}} b_{n, \lambda}(x-\lambda) \\
= & x b_{n, \lambda}(x-\lambda)-b_{n, \lambda}(x-\lambda) \\
& +2 \sum_{k=0}^{n} \sum_{m=0}^{k} \sum_{l=0}^{m}\left(\begin{array}{l}
n \\
k
\end{array}\right)\left(\begin{array}{c}
m \\
l
\end{array}\right) \lambda^{k-m+l} s_{1}(k, m) b_{n-k, \lambda}(-1)^{l} b_{m-l}(x) .
\end{aligned}
$$


Therefore, by (3.23), we obtain the following theorem.

Theorem 3.7. For $\mathrm{n} \geqslant 0$, we have

$$
\begin{aligned}
& b_{n+1, \lambda}(x)-x b_{n, \lambda}(x-\lambda)+b_{n, \lambda}(x-\lambda) \\
& =2 \sum_{k=0}^{n} \sum_{m=0}^{k} \sum_{l=0}^{m}\left(\begin{array}{l}
n \\
k
\end{array}\right)\left(\begin{array}{c}
m \\
l
\end{array}\right) \lambda^{k-m+l} s_{1}(k, m) b_{n-k, \lambda}(-1)^{l} b_{m-l}(x) .
\end{aligned}
$$

Remark 3.8. From $b_{n, \lambda}(x) \sim\left(2-e^{t}, \frac{1}{\lambda}\left(e^{\lambda t}-1\right)\right)$ and (2.6), we note that

$$
\begin{aligned}
\frac{d}{d x} b_{n, \lambda}(x) & =\sum_{l=0}^{n-1}\left(\begin{array}{l}
n \\
l
\end{array}\right)<\frac{1}{\lambda} \log (1+\lambda t) \mid x^{n-l}>b_{l, \lambda}(x) \\
& =\sum_{l=0}^{n-1}\left(\begin{array}{l}
n \\
l
\end{array}\right)<\sum_{m=1}^{\infty} \frac{(-\lambda)^{m-1} t^{m}}{m} \mid x^{n-l}>b_{l, \lambda}(x) \\
& =\sum_{l=0}^{n-1}\left(\begin{array}{l}
n \\
l
\end{array}\right)(-\lambda)^{n-l-1}(n-l-1) ! b_{l, \lambda}(x), \quad(n \geqslant 1) .
\end{aligned}
$$

Let

$$
\mathbb{P}_{n}=\{p(x) \in \mathbb{C}[x] \mid \operatorname{deg} p(x) \leqslant n\},(n \geqslant 0) .
$$

For $p(x) \in \mathbb{P}_{n}$, let

$$
p(x)=\sum_{l=0}^{n} a_{l} b_{l, \lambda}(x) .
$$

From (3.2), we note that

$$
<\left(2-e^{t}\right)\left(\frac{1}{\lambda}\left(e^{\lambda t}-1\right)\right)^{m} \mid b_{n, \lambda}(x)>=n ! \delta_{n, m},(n, m \geqslant 0) .
$$

By (3.24) and (3.25), we get for $0 \leqslant m \leqslant n$,

$$
\begin{aligned}
<\left(2-e^{t}\right)\left(\frac{1}{\lambda}\left(e^{\lambda t}-1\right)\right)^{m} \mid p(x)> & =\sum_{l=0}^{n} a_{l}<\left(2-e^{t}\right)\left(\frac{1}{\lambda}\left(e^{\lambda t}-1\right)\right)^{m} \mid b_{l, \lambda}(x)> \\
& =\sum_{l=0}^{n} a_{l} l ! \delta_{l, m}=a_{m} m !
\end{aligned}
$$

From (3.26), we have

$$
a_{m}=\frac{1}{m !}<\left(2-e^{t}\right)\left(\frac{1}{\lambda}\left(e^{\lambda t}-1\right)\right)^{m} \mid p(x)>,
$$

where $n \geqslant m \geqslant 0$. Therefore, we obtain the following theorem.

Theorem 3.9. For $\mathrm{p}(\mathrm{x}) \in \mathbb{P}_{\mathrm{n}}$, we have

$$
p(x)=\sum_{m=0}^{n} a_{m} b_{m, \lambda}(x),(n \geqslant 0),
$$

where

$$
a_{m}=\frac{1}{m !}<\left(2-e^{t}\right)\left(\frac{1}{\lambda}\left(e^{\lambda t}-1\right)\right)^{m} \mid p(x)>
$$


Let us take $p(x)=B_{n}(x) \in \mathbb{P}_{n}(n \geqslant 0)$. Then, by Theorem 3.9 we get

$$
B_{n}(x)=\sum_{m=0}^{n} a_{m} b_{m, \lambda}(x),(n \geqslant 0),
$$

where

$$
\begin{aligned}
a_{m} & =\frac{1}{m !}<\left(2-e^{t}\right)\left(\frac{1}{\lambda}\left(e^{\lambda t}-1\right)\right)^{m} \mid B_{n}(x)> \\
& =\frac{1}{m !}<\left(2-e^{t}\right) \lambda^{-m} m ! \sum_{k=m}^{\infty} S_{2}(k, m) \lambda^{k} \frac{t^{k}}{k !} \mid B_{n}(x)> \\
& =\sum_{k=m}^{n} \lambda^{k-m} S_{2}(k, m) \frac{1}{k !}<2-e^{t} \mid t^{k} B_{n}(x)> \\
& =\sum_{k=m}^{n} \lambda^{k-m} S_{2}(k, m)\left(\begin{array}{l}
n \\
k
\end{array}\right)<2-e^{t} \mid B_{n-k}(x)> \\
& =\sum_{k=m}^{n} \lambda^{k-m} S_{2}(k, m)\left(\begin{array}{l}
n \\
k
\end{array}\right)\left(2 B_{n-k}-B_{n-k}(1)\right) \\
& =\sum_{k=m}^{n} \lambda^{k-m} S_{2}(k, m)\left(\begin{array}{l}
n \\
k
\end{array}\right)\left(B_{n-k}-\delta_{1, n-k}\right) \\
& =\sum_{k=m}^{n} \lambda^{k-m} S_{2}(k, m)\left(\begin{array}{l}
n \\
k
\end{array}\right) B_{n-k}-n \lambda^{n-m-1} S_{2}(n-1, m)
\end{aligned}
$$

Therefore, by (3.27) and (3.28) we obtain the following theorem.

Theorem 3.10. For $\mathrm{n} \geqslant 0$, we have

$$
\begin{aligned}
B_{n}(x)= & \sum_{m=0}^{n} \sum_{k=m}^{n} \lambda^{k-m} S_{2}(k, m)\left(\begin{array}{l}
n \\
k
\end{array}\right) B_{n-k} b_{m, \lambda}(x) \\
& -n \sum_{m=0}^{n-1} \lambda^{n-m-1} S_{2}(n-1, m) b_{m, \lambda}(x) .
\end{aligned}
$$

For $b_{n, \lambda}(x) \sim\left(2-e^{t}, \frac{1}{\lambda}\left(e^{\lambda t}-1\right)\right),(x)_{n, \lambda} \sim\left(1, \frac{1}{\lambda}\left(e^{\lambda t}-1\right)\right)(n \geqslant 0)$, we have

$$
(x)_{n, \lambda}=\sum_{m=0}^{n} C_{n, m} b_{m, \lambda}(x),(n \geqslant 0),
$$

where

$$
\begin{aligned}
C_{n, m} & =\frac{1}{m !}<\left(2-e^{\frac{1}{\lambda} \log (1+\lambda t)}\right)^{-1}\left(\frac{1}{\lambda}\left(e^{\log (1+\lambda t)}-1\right)\right)^{m} \mid x^{n}> \\
& =\frac{1}{m !}<\left(2-(1+\lambda t)^{\frac{1}{\lambda}}\right)^{-1} t^{m} \mid x^{n}> \\
& =\left(\begin{array}{c}
n \\
m
\end{array}\right)<\sum_{k=0}^{\infty} b_{k, \lambda} \frac{t^{k}}{k !} \mid x^{n-m}> \\
& =\left(\begin{array}{c}
n \\
m
\end{array}\right) b_{n-m, \lambda} .
\end{aligned}
$$

Therefore, by (3.29) we obtain the following theorem. 
Theorem 3.11. For $\mathrm{n} \geqslant 0$, we have

$$
(x)_{n, \lambda}=\sum_{m=0}^{n}\left(\begin{array}{l}
n \\
m
\end{array}\right) b_{n-m, \lambda} b_{m, \lambda}(x) .
$$

The Korobov polynomials $K_{n, \lambda}(x)$ for $\lambda \neq 0,1$ are also defined in terms of generating function by

$$
\frac{\lambda t}{(1+t)^{\lambda}-1}(1+t)^{x}=\sum_{n=0}^{\infty} K_{n, \lambda}(x) \frac{t^{n}}{n !}, \quad(\text { see }[6]) .
$$

Thus, by (3.30), we get

$$
K_{n, \lambda}(x) \sim\left(\frac{e^{\lambda t}-1}{\lambda\left(e^{t}-1\right)}, e^{t}-1\right)
$$

For $K_{n, \lambda}(x) \sim\left(\frac{e^{\lambda t}-1}{\lambda\left(e^{t}-1\right)}, e^{t}-1\right), b_{n, \lambda}(x) \sim\left(2-e^{t}, \frac{1}{\lambda}\left(e^{\lambda t}-1\right)\right)$, we have

$$
K_{n, \lambda}(x)=\sum_{m=0}^{n} C_{n, m} b_{m, \lambda}(x),(n \geqslant 0),
$$

where

$$
\begin{aligned}
C_{n, m} & =\frac{1}{m !}\left\langle(1-t) \frac{\lambda t}{(1+t)^{\lambda}-1}\left(\frac{1}{\lambda}\left((1+t)^{\lambda}-1\right)\right)^{m} \mid x^{n}\right\rangle \\
& =\frac{\lambda^{-m}}{m !} \sum_{l=0}^{m}\left(\begin{array}{c}
m \\
l
\end{array}\right)(-1)^{m-l}\left\langle(1-t) \frac{\lambda t}{(1+t)^{\lambda}-1}(1+t)^{l \lambda} \mid x^{n}\right\rangle \\
& =\frac{\lambda^{-m}}{m !} \sum_{l=0}^{m}\left(\begin{array}{c}
m \\
l
\end{array}\right)(-1)^{m-l}\left\langle(1-t) \mid \sum_{j=0}^{n} K_{j, \lambda}(l \lambda) \frac{t^{j}}{j !} x^{n}\right\rangle \\
& =\frac{\lambda^{-m}}{m !} \sum_{l=0}^{m}\left(\begin{array}{c}
m \\
l
\end{array}\right)(-1)^{m-l} \sum_{j=0}^{n} K_{j, \lambda}(l \lambda)\left(\begin{array}{c}
n \\
j
\end{array}\right)\left(\delta_{j, n}-\delta_{j, n-1}\right) \\
& =\frac{\lambda^{-m}}{m !} \sum_{l=0}^{m}\left(\begin{array}{c}
m \\
l
\end{array}\right)(-1)^{m-l}\left(K_{n, \lambda}(l \lambda)-n K_{n-1, \lambda}(l \lambda)\right) .
\end{aligned}
$$

Therefore, by (3.31) and (3.32), we obtain the following theorem.

Theorem 3.12. For $n \geqslant 0$, we have

$$
K_{n, \lambda}(x)=\sum_{m=0}^{n}\left(\frac{\lambda^{-m}}{m !} \sum_{l=0}^{m}\left(\begin{array}{c}
m \\
l
\end{array}\right)(-1)^{m-l}\left(K_{n, \lambda}(l \lambda)-n K_{n-1, \lambda}(l \lambda)\right)\right) b_{m, \lambda}(x) .
$$

\section{References}

[1] E. T. Bell, Postulational bases for the umbral calculus, Amer. J. Math., 62 (1940), 717-724. 2

[2] L. Carlitz, Degenerate Stirling, Bernoulli and Eulerian numbers, Utilitas Math., 15 (1979), 51-88. 1.2, 1

[3] L. Comtet, Advanced Combinatorics: The Art of Finite and Infinite Expansions, D. Reidel Publishing, Holland, (1974). $1.3,1.5$

[4] R. Dere, Y. Simsek, Applications of umbral algebra to some special polynomials, Adv. Stud. Contemp. Math., 22 (2012), 433-438. 1

[5] A. Di Crescenzo, G.-C. Rota, On umbral calculus, Ricerche Mat., 43 (1994), 129-162. 2

[6] D. V. Dolgiř, D. S. Kim, T. Kim, Korobov polynomials of the first kind, Sb. Math., 208 (2017), 60-74. 1, 1, 2.3, 3.30 
[7] T. Kim, Identities involving Laguerre polynomials derived from umbral calculus, Russ. J. Math. Phys., 21 (2014), 36-45. 1,2

[8] D. S. Kim, T. Kim, J. J. Seo, Higher-order Daehee polynomials of the first kind with umbral calculus, Adv. Stud. Contemp. Math., 24 (2014), 5-18. 1, 1, 2, 2.3

[9] S. Roman, The theory of the umbral calculus, J. Math. Anal. Appl., 95 (1983), 528-563. 2.2

[10] S. Roman, The umbral calculus, Academic Press, New York, (1984). 1, 2, 2.2, 2, 2.3, 2.4, $2.5,2$

[11] G. C. Rota, B. D. Taylor, An introduction to the umbral calculus, Analysis, geometry and groups: a Riemann legacy volume, Hadronic Press, Palm Harbor, (1993). 2.5

[12] G. C. Rota, B. D. Taylor, The classical umbral calculus, SIAM J. Math. Anal., 25 (1994), 694-711. 2 